\title{
Multiple Systems Estimation Techniques for Estimating Casualties in Armed Conflicts*
}

\author{
Daniel Manrique-Vallier; Megan E. Price $\ddagger$ and Anita Gohdes ${ }^{\S}$
}

February 17, 2012

\begin{abstract}
We offer an introduction to the logic of using multiple systems estimation (MSE) in the context of estimating casualties in armed conflict. We demonstrate how this estimation technique relies on four strong assumptions, which are typically explored and adjusted for with the generalization to three or more systems and a variety of MSE methods and estimators. We find that the implications of violating these four classic assumptions, in particular the homogeneity and independence assumptions, do not translate clearly to the more-than-three-systems case. We use case studies from human rights research to present the subtle ways that misunderstanding these assumptions can lead to misapplication and misinterpretation of MSE methods. Additionally, we examine how MSE methods rely on patterns of inclusion or capture to represent the underlying population of interest even when the individual samples themselves are not representative.
\end{abstract}

\footnotetext{
${ }^{*}$ Working Paper submitted for publication in an edited volume on casualty estimation in times of conflict. Comments are welcome.

${ }^{\dagger}$ Social Science Research Institute and Department of Statistical Science, Duke University

${ }^{\ddagger}$ Benetech Human Rights Data Analysis Group

${ }^{\S}$ University of Mannheim and Benetech Human Rights Data Analysis Group. Corresponding author: Anita.Gohdes@uni-mannheim.de
} 


\section{Introduction}

During and after armed conflicts, different groups attempt to gather information on the extent to which violence has claimed human lives. Depending on the reasons why the group or institutions record this information, lists of casualties are compiled with more or less detailed data and coverage. For example, humanitarian organizations collect information on cases known to them, death registries try keep track of the deceased, and press agencies report on victims fallen in battle. Retrospectively, it is often national Truth Commissions and human rights non-profit organizations that document atrocities committed, in order to help recollect the past and provide justice to the victims of conflict.

All of these 'casualty lists' are prone to incomplete registration, be it for institutional, financial, geographical or political reasons. Answers to questions about the real magnitude and characteristics of the conflict cannot be obtained from any single 'found' data source in a direct way. However, with basic infrastructure and security oftentimes lacking in conflict or post-conflict settings, researchers and practitioners attempting to determine the actual number of casualties that resulted from the conflict commonly find themselves in a situation where they have to rely on these data sources as the basis of their inquiries. Statistical methods that enable to draw conclusions about the entire population based on those incomplete data sources are thus desirable.

This paper offers an introduction to one such statistical tool, Multiple Systems Estimation (MSE) methods. More specifically, for the estimation of casualties in armed conflict, these methods provide a way to quantify the probability that a death will be missed, i.e. that no enumeration effort will record it, and therefore, a way to estimate the undercount. MSE methods comprise a rather broad family of statistical techniques specifically designed to estimate undercounts in situations like the one described here, where multiple intersecting, but incomplete, lists are available. They offer an opportunity to take advantage of the multiplicity of data sources often found in armed-conflict situations for drawing conclusions about the underlying extent of the conflict they document.

These methods date back at least to the 19th century, when they were developed to estimate the size of animal populations. ${ }^{1}$ For this reason much of the language associated with MSE methods often refer to 'captures,' such as 'capture probabilities.' This is carried over from studies in which animals were captured, tagged, and released (Petersen, 1896). Since then they have been adapted to deal with human populations, in applications that range from

\footnotetext{
${ }^{1}$ See Goudie and Goudie (2007) for an interesting account of the origin of these techniques
} 
census undercount correction (Sekar and Deming, 1949), problems in epidemiology (Hook and Regal, 1999; for Disease Monitoring and Forecasting, 1995a,b), and casualty estimation (Ball et al., 2002, 2003), among others. Due to the development of these methods across a variety of fields, a variety of terminology has also developed to describe essentially the same class of methods. Besides MSE, these methods are also known as - among other names - Multiple Recapture Estimation, Multiple-Record Systems Estimation and, in the particular case of two systems, Capture-Recapture and Dual Systems Estimation. While we have aimed for consistency favoring the name MSE (the preferred term for the method applied to human populations), all of them may be used interchangeably.

We begin with an overview of the statistical intuition that underlies MSE methods, as it has some particularities that set it apart from more traditional and well known statistical techniques. Section 2.1 deals with the two-list case, which is then developed into a general multilist framework in Section 2.2. Section 2.2.1 provides a deeper reflection on two of the classic assumptions of the basic two-list model, and the challenge of interpreting and testing these assumptions in the general case. We address the question of representing unobserved individuals in Section 2.2.2. In some sections we occasionally rely on mathematical notation to refer back to concepts that otherwise would require lengthy - and ambiguous - prose to describe. While comfort with mathematical notation and basic probability theory is beneficial, it is not indispensable to understand this chapter. Finally, we present two case studies in Sections 3.1 and 3.2, from Kosovo and Peru, to further illustrate applications, challenges, and successes of MSE techniques. The paper concludes with a discussion of the opportunities and limitations that these methods offer to the field of casualty estimation in armed conflicts .

\section{Basics of Multiple Systems Estimation}

As mentioned in the introduction, any effort to enumerate casualties will likely miss some individuals. Certain geographic areas may be too remote to access or still too violent and unstable for researchers to safely collect data. In some areas wide-sweeping violence may not leave behind any witnesses to tell researchers about what happened, or existing witnesses may choose not to tell their story.

In general, multiple systems estimation (MSE) methods attempt to estimate the number of cases that were not included in lists that partially enumerate a closed population. In this context, consider a conflict situation, where an unknown number, $N$, of individuals were killed. Now, assume that different 'counting teams', working independently, have already attempted to enumerate them. Each team will have counted a part of the casualty population; some of 
the individuals will have been counted by more than one team, and some will not have been counted at all.

If we had access to all these lists, the first thing we could try is to pool all of them into a single comprehensive list. Since some individuals will have been counted more than once, and some left out, it is very likely that the combination of all lists will also be incomplete. If the teams recorded some identifying information on the individuals, we can remove the duplicates by comparing the datasets and noting who and how many of those counted were included on more than one list. As we will see, this inclusion, or capture pattern, i.e. which lists included and which missed an individual, is the crucial piece for the MSE calculations. For now, we can safely state that the de-duplication (i.e., identification and removal of duplicates) of individuals who were included on one or more lists gives us a lower bound on the total size of the population of interest, assuming that de-duplication efforts were successful. The question that remains is: 'How many individuals were not counted by any of the teams?'

Table 1 shows an example of such a de-duplication and matching of different lists into one dataset. ${ }^{2}$ Every individual counted now only appears in one row, with the last three columns containing the information on which list recorded the case. The example given here demonstrates how binary information indicating included (1) or not included (0) in list A, B or C creates an 'inclusion pattern'. The diagram in Figure 1 presents the same information in a graphical form. Note how each inclusion pattern univocally refer to a location in the Venn diagram. ${ }^{3}$ Again, each individual appears just once in the diagram which informs us about their corresponding inclusion pattern. Inclusion patterns represent the link between the concepts of "unique individual" and 'records on a list'.

MSE techniques attempt to provide an answer to the question of who was not included in any of the lists by looking at this inclusion pattern for each individual that was included. We start by modeling the probability that an individual was reported on only a single list. Then, we can also model the probability that an individual was reported on two specific lists. Building on this, we can build up a model that describes the entire set of possible patterns of multiple reporting of the same individual(s), including the one that corresponds to 'no report'.

\footnotetext{
${ }^{2}$ This is a fictitious table that was created for exemplary purposes. In real-life cases, the information existing for each individual varies profoundly. The co-variables included here (age, sex, location) merely describe some of the most frequently encountered victim/incidence characteristics.

${ }^{3}$ Since Table 1 only shows four individuals, not all inclusion patterns are populated in the diagram. A complete list could potentially include individuals captured in all locations of the diagram, except outside all circles.
} 
Table 1: Example: 'clipping' of a matched and de-duplicated list of casualties

\begin{tabular}{|r|r|r|r|r|r|r|r|}
\hline ID & Sex & Age & Location & Date & List A & List B & List C \\
\hline.. &.. &.. &.. &.. &.. &.. &.. \\
\hline 948 & M & 32 & south-west & 1999 & 0 & 1 & 0 \\
\hline 949 & M & 45 & west & 1995 & 1 & 1 & 0 \\
\hline 950 & $\mathrm{~F}$ & 30 & south-west & 1990 & 0 & 0 & 1 \\
\hline 951 & M & $?$ & west & 1991 & 1 & 1 & 0 \\
\hline.. &.. &.. &.. &.. &.. &.. &.. \\
\hline
\end{tabular}

Figure 1: Schematic representation of de-duplicated list in Table 1.

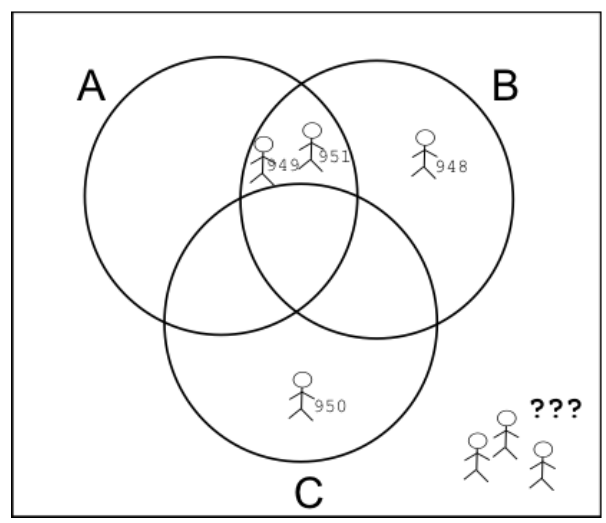

Intuitively, it is easy to see how inclusion pattern information can help us to learn more about the unknown size of the population. Let us assume that one of the teams in our example did a particularly thorough job and the list it produced is already close to a full enumeration. In such a case, any additional comparable list by another team is likely to have a considerable number of individual records in common with the first list. Conversely, if a team only covered a very small part of the actual population of interest, an additional similar list can only be expected to share just a few individual records with it. Note that if a list were actually a full enumeration, any additional list can only be a subset and will thus only repeat individuals already recorded.

If we formalize the relationships between the probabilities of these inclusion patterns, then we can - with the help of some additional assumptions - estimate the probability distribution of multiple inclusions in lists, and thus ultimately estimate the probability of an individual not being included in any of the lists. The following section illustrates this procedure in the two-list case. 


\subsection{Classic Two-Systems Estimation}

Consider the number of casualties in a conflict to be $\mathrm{N}$-more generally, $\mathrm{N}$ is the unknown size of a finite population of interest. We assume that we have available two incomplete lists, A and $\mathrm{B}$, that enumerate some of the individuals in the population. If we consider an arbitrary individual, he or she will necessarily fall into one of the following four cases: the individual is included in the first list, but not in the second (this case will be represented by $O_{10}$ for the remainder of this chapter), or, in the second list, but not in the first $\left(O_{01}\right)$, or in both of the lists $\left(O_{11}\right)$. Finally, the individual might not be included in either list $\left(O_{00}\right)$. We call these cases inclusion patterns.

It is important to note that although every single individual in the population must fall into one (and only one) of these categories, we only have information on the individuals that were actually included in at least one of the lists. We can thus only observe the individuals that correspond to the first three inclusion patterns, $O_{10}, O_{01}$ or $O_{11}$.

Now consider an arbitrary individual, $i$, from the population. ${ }^{4}$ We can assign a probability to the event that any one individual $(i)$ falls into each of the categories described above. For example, we can consider the probability that individual $i$ is on list A but not B. As defined above, this category is represented as $O_{10}$. We denote this probability $p_{10}^{i}=P$ (Individual $\mathrm{i}$ in category $O_{10}$ ). We can then associate each individual with a 'list' (a 4-dimensional probability vector) of their probability of falling into each of the four inclusion patterns. This vector, $\left(p_{00}^{i}, p_{01}^{i}, p_{10}^{i}, p_{11}^{i}\right)$, fully describes the probabilities of individual $i$ being documented (or not) according to each possible inclusion pattern.

As is common when using a statistical model to describe a real-world situation, some assumptions are necessary to estimate the quantities of interest. As the simplest version of this method, the two-list situation requires very strong — and untestable - assumptions. We will revisit them in the next section in the context of the existence of multiple lists.

While not the only possibility, the usual assumptions in the two-list case are

1. Closed System: The lists refer to a closed system: N must refer to the same population in each dataset. ${ }^{5}$

\footnotetext{
${ }^{4} i$ could equal any number between 1 and $N$, indicating the first, second, etc. individual in the population; in mathematical notation we write $i=1, \ldots, N$.

${ }^{5}$ For the case discussed here, this assumption is generally met: individuals that were killed in armed conflict add to the number of casualties and cannot 'leave' this population.
} 
2. Homogeneity: For each list, every individual must have the same probability of being included, or captured. ${ }^{6}$

3. Independence: The probability that an individual is or is not included on list A is not affected by being included (or not) on list B, and vice versa.

Intuitively, the homogeneity assumption refers to the fact that no individual should be intrinsically "more listable" than the others due to individual traits. Independence, in turn, requires lists that have been created without influencing one another (i.e., inclusion on one list does not affect the likelihood of inclusion on another list). Going back to the example given above, these conditions require that (1) no victim has any distinctive characteristic (e.g. age) that makes her more likely than the others to be in any list, and (2) each team worked without receiving information from any other team. As can be seen, both the homogeneity and the independence assumptions pose high demands to the data, demands that are unlikely to be fulfilled - even approximately — in the casualty estimation context. ${ }^{7}$ However, as is the case with other statistical methods, there are means by which we can detect departures from and alternatively account for these assumptions. We will discuss this in more detail in Section 2.2.1.

To estimate the unknown population size, the above assumptions must be mathematically formalized and combined with some statistical technique that allows the calculation of such numbers (an "estimator", in statistical terminology). To illustrate this process, the rest of this section shows the derivation of one specific two-list estimator, the Petersen Estimator, which is the best known of the two-list estimators (Petersen, 1896; Bishop, Fienberg and Holland, 1975; for Disease Monitoring and Forecasting, 1995a). Readers without elementary knowledge of probability and statistics can safely skip the rest of this section.

Elementary probability calculations show that the above stated assumptions have two immediate consequences. First, the same set of pattern probabilities apply to every individual — this is, we can drop the index $i$ in the probability vector, making $\left(p_{00}^{i}, p_{01}^{i}, p_{10}^{i}, p_{11}^{i}\right)=\left(p_{00}, p_{01}, p_{10}, p_{11}\right)$. Second, these probabilities must satisfy the condition $p_{01} p_{10} / p_{11} p_{00}=1$. These two conditions, together with the fact that the population is finite, define a model for the distribution of capture probabilities. Standard statistical techniques can then be used to estimate the parameters of the model, including the population size.

\footnotetext{
${ }^{6}$ This definition of homogeneity is simple and appropriate in this two-list context. In more complex settings, however, it can be too restrictive to be really useful. A more general definition has to be stated with respect to a model: the recording of each individual can be well described by the same model.

${ }^{7} \mathrm{~A}$ fourth assumption usually made is perfect matching: Each of the included individuals must be perfectly categorized to be either only included in system A, only included in system B, or included in both system A and B (see Lum et al., 2010, 3)).
} 
One simple way of obtaining the Petersen estimator is the following. Under the proposed model, the expected values of the number of individuals in each capture category is $m_{a b}=$ $E\left[n_{a b}\right]=N \cdot p_{a b}(a=0,1 ; b=0,1),{ }^{8}$ where $n_{a b}$ are the actual (observed and unobserved) counts, $N$ is the true (unknown) population size, and $p_{a b}$ is the general form of the probabilities defined above. Then, the condition above that $p_{01} p_{10} / p_{11} p_{00}=1$ can be rewritten as

$$
m_{00}=\frac{m_{01} m_{10}}{m_{11}} .
$$

We can use the observed counts as estimators for their expected values, $\hat{m}_{01}=n_{01}, \hat{m}_{10}=n_{10}$ and $\hat{m}_{11}=n_{11}$ and use them to estimate the undercount:

$$
\hat{n}_{00}=\frac{\hat{m}_{01} \hat{m}_{10}}{\hat{m}_{11}}=\frac{n_{01} n_{10}}{n_{11}} .
$$

The Petersen estimator or the dual-system estimator is the best known of the two-system population size estimators and is often used illustrate the core Capture-Recapture ideas. It is usually motivated as an extrapolation of capture ratios through a process that implicitly requires the independence and homogeneity assumptions (see e.g. for Disease Monitoring and Forecasting $(1995 a)$ ). It can also be shown to be a conditional Maximum Likelihood estimate for the two-variable log-linear independence model (Fienberg, 1972; Bishop, Fienberg and Holland, 1975).

\subsection{Multiple Systems Estimation (MSE): The general case}

As we have just seen, MSE techniques draw inferences about the size of the population of interest from the classification of observed (listed) individuals into multiple inclusion patterns. In the case of 2 lists, there are only 4 possible classifications or 'partitions', of which one, $\mathrm{O}_{00}$, is unobservable. With only three partitions, the analyst has very limited information on which to base her estimations. This is why strong assumptions like those stated in the previous section are unavoidable.

More than two lists, in contrast, provide a much richer set of information from which we can draw inferences. Since every additional list has the effect of increasing the number of observable patterns exponentially, we gain observable information, while the unobservable pattern (individuals not included in any list) remains fixed at one. For instance, 3 lists produce $2^{3}-1=7$ observable partitions $\left(O_{001}, O_{010}, O_{011}, O_{100}, O_{101}, O_{110}\right.$ and $\left.O_{111}\right)$, while 15 lists (see e.g. Lum et al. (2010)), produce $2^{15}-1=32,767$ observable partitions. In both

\footnotetext{
${ }^{8}$ The subscripts $a b$ are a shortcut to refer to any of the four inclusion patterns: $00,01,10,11$.
} 
cases, we have only one unobservable pattern $\left(O_{000}\right.$ and $O_{000000000000000}$, i.e., those that were missed by all three and all 15 lists respectively).

An increasing number of partitions offer more information, which enables the use of more sophisticated models that rely on weaker assumptions than the ones described for the twosystem model. Unfortunately, the modeling of that increasing number of inclusion patterns also requires increasingly sophisticated statistical techniques.

Several families of models have been proposed to deal with the multiple-list situation, each of which specifies different sets of assumptions about the population and the list creation processes. For example, log-linear models (Fienberg, 1972; Bishop, Fienberg and Holland, 1975) take advantage of multiple lists to account for the effect of list interdependence, by modeling dependence patterns explicitly. Other techniques, that respond to different assumptions, include Bayesian versions of standard approaches (George and Robert, 1992), latent class models (Basu and Ebrahimi, 2001), Grade of Membership models (Manrique-Vallier and Fienberg, 2008), and Rasch models (Fienberg, Johnson and Junker, 1999), to name just a few.

A generalization of the framework from the two-list case to the multi-list case is straightforward: if we have a number, $J$, of lists, then any individual in the population can be classified into one and only one inclusion pattern $O_{X_{1} X_{2} X_{3} \ldots X_{J}}$, where $X_{j}=1$ indicates presence in list $j$ and $X_{j}=0$, absence. For each individual, $i$, we can then model the probability distribution over the inclusion patterns. The information about the $2^{J}-1$ observable patterns is then used to estimate the probability of the single unobserved pattern, $O_{000 \ldots .000}$, for those unobserved individuals. The specific way this information is used to estimate the probability of the single unobserved pattern varies across the general class of MSE methods.

In addition to allowing weaker - and therefore, more realistic - assumptions, the use of more than two lists also provides the means to test the plausibility of such assumptions. This feature is crucial since successful estimation of the unobserved pattern depends on accurately modeling the inclusion-exclusion pattern present in the population. Accurate models rely on appropriately chosen methods, based on which assumptions are met (or not) by the observed data. This presents an additional challenge since observed data must be examined to determine the most appropriate method, and in many cases, a chosen method may still result in several plausible models. When a set of inclusion patterns appears to be adequately described by more than one model, a 'best' model may be chosen (an example of this can be found in the Kosovo case study in Section 3.1) or estimates may be based on an average of multiple 
models (see Lum et. al. 2010). ${ }^{9}$

It is also important to keep in mind that testing the plausibility of the assumptions listed above can only be based on the observed data (Fienberg, 1972; Bishop, Fienberg and Holland, 1975; for Disease Monitoring and Forecasting, 1995a). This means that no matter how well the observable patterns are modeled, we can only estimate the number of unobserved individuals through the untestable assumption that the inclusion pattern of those unobserved individuals (more precisely, their lack of inclusion) can be described by the same model that describes the inclusion pattern of those we get to observe. This is an inescapable limitation that must be taken seriously. We will elaborate on this idea in Section 2.2.2.

\subsubsection{A Closer Look at Heterogeneity and Dependence}

Homogeneity and independence are intuitive assumptions that are sometimes reasonable for specific applications, such as some particular estimations of wildlife populations. However, in more sophisticated settings, such as casualty estimation, we cannot expect them to hold.

In general, victims of violence are likely to be a heterogeneous group, and lists describing them are unlikely to be independent. People have different social visibility due to networks, geographic location and even occupation. These characteristics, which often influence the outcome to some extent, are called covariates in statistics ${ }^{10}$. Different documentation projects have different objectives and may have different propensities to record victims with particular characteristics/covariates. Projects will sometimes collaborate or share information with each other, directly inducing dependence between lists. ${ }^{11}$

In cases where covariate information on the particularities of the victim and context is available, stratification can be used as a means to reduce the effect of heterogeneity. In short, stratification works by partitioning the population into separate and relatively more homogeneous subsets, where the modeling assumptions can be expected to hold better. ${ }^{12}$ Estimates are then calculated within each stratum, using the chosen MSE method. For example, if we think that the place of death can strongly influence capture probabilities (i.e., that it is an

\footnotetext{
${ }^{9}$ The 'model selection' problem is a major subject in statistical methodology, common to a wide range of applications. Although it is a crucial problem in MSE applications, due to space constraints we will not elaborate it further.

${ }^{10}$ Examples of covariates include sex, age, location, and date from Table 1 above

${ }^{11}$ Although this may seem a pervasive problem, in actual applications, the situation is not as dire. For instance, in the Peruvian study (Ball et al., 2003), shared information was clearly labeled and could be separated without problem. As we will see when discussing the applications (Sections 3.1 and 3.2), thorough knowledge of the data is essential.

${ }^{12}$ In other words, where the inclusion patterns can be described reasonably well by a particular model.
} 
important covariate), as is likely to be true in many cases, we can divide the combined sample into geographic subgroups. More precisely, introducing a stratification scheme is equivalent to assuming that, if two individuals, $i$ and $j$, belong to the same stratum (e.g. the same geographic region), then the probability of each pattern of inclusion is the same for both or, more formally, $\operatorname{Pr}\left(\right.$ individual $\mathrm{i}$ in $\left.O_{X}\right)=\operatorname{Pr}\left(\right.$ individual $\mathrm{j}$ in $\left.O_{X}\right)$.

When relevant covariate information is not available, the only visible effect of heterogeneity is the appearance of dependences between lists. Take for example the case of a conflict with two main perpetrators and three available lists documenting casualties, where no list registered information about the perpetrator of each casualty. If two lists were more prone to register people that were victims of a particular perpetrator, while the other one proceeded in a more balanced way, then - assuming for now that no other sources of heterogeneity are as important - the observable effect will be the emergence of (positive) dependence between the first two lists, while the third will remain relatively independent. In cases like these, with no covariate information that could fully explain it, the only way we can learn about the heterogeneity structure is through the induced dependencies.

An important consequence of the above is that in many cases the observable dependence between lists can provide enough information to successfully account for the effects of the heterogeneity. Furthermore, sometimes accounting for the induced dependence amounts to directly controlling the effects of the heterogeneity. For example, some general patterns of heterogeneity can be successfully represented or approximated by interactions between lists with the aid of log-linear models. ${ }^{13}$

\subsubsection{Representing Unobserved Individuals}

As we have seen, the ultimate goal of MSE techniques is to estimate the undercount. This requires estimating probabilities of non-inclusion in lists, which relies on the information about non-inclusion contained in the inclusion patterns of those individuals observed at least once. To understand this apparent paradox, it must be noted that in most cases, many individuals in a combined J-list sample are likely to have been missed by one or more lists. This means that the observable inclusion patterns contain a great deal of information about individuals who are not included on a given list. For instance, if we had six lists, we would have $2^{6}-1=63$ observable inclusion patterns, from which 62 of them describe ways of not

\footnotetext{
${ }^{13}$ In general, heterogeneity that affect groups of lists, but not every list, can be directly accounted for as interactions between lists in log-linear models. Other, more sophisticated, patterns that simultaneously affect all lists can also have estimable log-linear representations (see e.g. Darroch et al. (1993) for more details).
} 
being on lists. ${ }^{14}$ Not being in all lists simultaneously is just one more way of not being in lists.

As mentioned above, the arguably most basic assumption in MSE is that the non-inclusion of the fully unobserved individuals (those not included in any list) can be represented by the same model that represents the inclusion (and non-inclusion) of those we get to observe in at least one list. While this is a strong and untestable condition, we argue that it is far less demanding that it may seem at first glance.

To better understand this requirement, let us examine its violation. Assuming that nonobserved individuals (those with inclusion pattern $O_{0 . .0}$ ) differ substantially from observed ones (those with all the other $2^{J}-1$ patterns), amounts to assuming that the event of simultaneously not appearing in all those particular lists is somehow an intrinsic attribute of those individuals. This means, for example, that being missed by five lists but not by a sixth, in a six-list case, is qualitatively different from being missed by those six lists; and that, if we added another seventh list, being missed by all previous six lists but not by the new one is also substantially different from being missed by all seven. Except for a few situations, it appears to us more difficult to reject this requirement than to accept it.

One of these problematic situations is the case of erroneously assumed coverage. This would, for instance, occur if we had a situation where our lists were specifically designed so that they only reported events on a particular region - and ignored any other report - but we assumed we obtained estimates for a wider region. ${ }^{15}$ This would lead to the existence of two classes of subjects: those who are, at least in principle, listable and those who are not. In the language of capture probabilities, the first group have a positive probability of inclusion, while for the second, that probability is exactly zero. Any individual who has an intrinsic attribute which causes him or her to be unobserved by any list has, by definition, a capture probability of zero. Individuals with capture probabilities of zero cannot be represented by any data collection mechanism. In contrast, individuals with non-zero capture probabilities, who just happen to be un-observed by every list (not due to some intrinsic attribute but rather by chance) are likely to be represented by other, observed individuals (who are also missed by some subset of the lists).

Related to concerns regarding how best to represent unobserved individuals is the belief that MSE techniques can only produce valid inferences when based on lists that are random

\footnotetext{
${ }^{14}$ As a further example, the observable pattern $O_{000100}$ in a 6-list situation not only gives us information about being in the fourth list, but also about not being in lists 1, 2, 3, 5 and 6 .

${ }^{15}$ This situation is analogous to a survey with a sampling frame that does not cover the totality of the target population.
} 
samples from the target population (e.g. Jewell et. al. in this volume). This point is of particular importance, since arguably most lists of casualties that can be found in practice are unable to meet such a standard. Fortunately, except in some truly problematic cases, this is not correct. The key is that the only information that matters for MSE estimation is the relationship between lists, and not the lists' composition. It can be shown that all that is required is that the collection of inclusion patterns is representative of the relationships between the lists, not for each list to represent the underlying population. As an extreme example, consider an organization that collected information giving preference to individuals that lived close to their headquarters, and another one that did so giving preference to older people over younger people. This is an example where none of the partial samples is representative of the characteristics of the target population and where the "homogeneity" assumption, understood as the same probability of inclusion within each list, is clearly violated. However, if we assume, as seems reasonable, that age is uncorrelated with how close to the organization headquarters the victim lived, we can show that even the simple 2-list Petersen estimator is valid. The example is, of course, artificial, ${ }^{16}$ but serves well to illustrate the point. As long as we can approximately model the characteristics of the resulting aggregated pattern-independence in the example - the internal characteristics of the lists turn out to be irrelevant. ${ }^{17}$

Real complications may arise, however, if the underlying data structure is such that a "wrong model" can successfully account for the observable part of the inclusion patterns, but not for the full-exclusion one $\left(O_{0 \ldots 0}\right)$, and we are led to choose it over other more appropriate ones. In theory, some heterogeneity patterns could lead to such a situation. For example, Jewell et. al. in this volume present one extreme situation, with lists from distinct age groups with little or no overlap - where they correctly conclude that most MSE procedures would considerably overestimate the actual total counts ${ }^{18}$. This may be an example of a heterogeneity pattern in the population that could plausibly induce a pattern of dependences between lists that would be consistent with an identifiable model for all the $\left(2^{J}-1\right)$ observable patterns, but somehow not for the unobserved category $O_{000 \ldots 000}$. In this general case, the risk is that we would be led to accept and rely on a model that does not correctly represent the unobserved individuals and therefore poses a risk for producing biased estimates. However, it is not well understood which plausible patterns of heterogeneity can induce such outcomes and more research is needed on this topic. In the authors' experience, populations where the same source of heterogeneity strongly affects all lists simultaneously sometimes can generate observable

\footnotetext{
${ }^{16}$ And, depending on the specific situation at hand, it could also be argued that the age distribution could be correlated with geographic location.

${ }^{17}$ This is one reason why we believe that understanding homogeneity as equal probability of being in each list can be misleading in this discussion.

${ }^{18}$ see also Bishop, Fienberg and Holland (1975) Ch. 6 for a discussion about essentially the same extreme situation
} 
data with these characteristics.

However, while acknowledging such extreme situations as real limitations, we should bear in mind that, although plausible, they are unlikely be completely unknown to researchers. In the example, for instance, a simple tabulation of the counts broken down by age would immediately reveal this special heterogeneity. This would allow researchers to stratify the estimations, in this example, by age group. Moreover, if researchers were able to secure any other list that was less sensitive to that particular source of heterogeneity - even if it was extremely sensitive to any other uncorrelated source - such list could potentially provide enough information to overcome the problem through direct modeling of the dependence patterns - in theory, even without stratification.

As will be shown in more detail for the case of Peru (below), the researchers' knowledge of the situation is crucial here. In Peru, two of the lists used for the three-system estimation of killed and disappeared people across the 20 year conflict gravely underreported acts committed by the rebel group Sendero Luminoso. With the help of the third, largest, list provided by the Truth and Reconciliation Commission, the research team was able to use the information provided by all three lists to account for this 'perpetrator heterogeneity' and estimate credible levels of casualties committed by both the state and rebel group.

\section{Case Studies}

\subsection{Kosovo}

The tragic events that unfolded in Kosovo between March and June 1999 present a case where the application of MSE methods significantly improved the knowledge of the extent of violence that was exercised against Kosovars. The research was conducted by Ball et al. (2002), who used four data sources to conduct MSE analyses of the patterns of refugee flow and killings in Kosovo for said time period. The data sources comprised of interviews conducted by the American Bar Association Central and East European Law Initiative (ABA/CEELI), interviews conducted by Human Rights Watch (HRW), interviews conducted by the Organization for Security and Cooperation in Europe (OSCE), and records of exhumations conducted on behalf of the International Criminal Tribunal for the Former Yugoslavia (ICTY). It is important to note that while excellent sources of information, and exemplary data collection efforts, none of these sources are uniformly representative of the entire underlying population of individuals killed in Kosovo between March and June 1999. For example, ABA/CEELI conducted interviews in Macedonia (among other locations) relying on referrals from humanitarian organizations, word of mouth, advertising in local newspapers, and moving tent by 
tent through refugee camps (Ball et. al. 2002). These were reasonable methods to locate individuals with crucial information on killings in Kosovo. Similar methods are employed in a variety of conflict and post-conflict regions and are often necessary to obtain information where there is no hope of a complete census or a sampling frame from which to build a random sample. But it would be unreasonable to assume that these methods result in a representative sample. Therefore we must rely on statistical methods, such as MSE, which are suitable for calculating population-level estimates from available data.

A total of 4,400 individual victims were identified across the four data sources. Many of these were listed in more than one source. Based on the inclusion patterns of these 4,400 identified individuals, an estimated total of 10,356 victims was calculated (95\% confidence interval: $[9,002,12,122])$. This number was surprising, as it implies that more victims went undocumented - namely, 5,956 — than were jointly recorded in the four available data sources.

Prior to building and selecting the models necessary to calculate these estimates, exploratory data analysis was conducted to evaluate the plausibility of the classic MSE assumptions outlined in Section 2.1. This analysis indicated potential sources of heterogeneity, and led researchers to stratify MSE calculations by space (geographic region) and time. Two-systems estimates (as described in Section 2.1) were also calculated for each pair of sources to identify possible dependences between lists; numerous positive dependences were identified. An extension of this method, using hierarchical log-linear models, was used to examine the relationships between three of the data sources at a time. ${ }^{19}$ These results indicated that the pairwise dependencies, identified by the two-systems estimates, were likely well modeled by including two-way interaction terms. Such direct analysis of the data patterns (over time and space) and exploratory two- and three-systems MSE calculations indicated the need for careful stratification and complex modeling to account for the intricate heterogeneity and dependence structure. This procedure illustrates how, as mentioned in Section 2.2, assumptions must be checked so that the most appropriate MSE method is chosen for a given situation.

There were many possible complex models to describe the observed inclusion patterns. Traditional model selection techniques (i.e., best fit statistics) were used to identify the model used to calculate 10,356 killings with a $95 \%$ confidence interval of $(9,002,12,122)^{20}$.

It is important to note a few things. First, without MSE calculations, at that time, we would have lacked an estimate of the nearly 6,000 undocumented killings in Kosovo between

\footnotetext{
${ }^{19}$ See Bishop, Fienberg and Holland (1975) for details on hierarchical log-linear models.

${ }^{20}$ See (Ball et al., 2002) for full analytical methods and results, including model results calculated for space and time strata.
} 
March and June 1999. Second, if we relied solely on the observable, available data from the four sources, we would be unable to choose between the contradictory conclusions regarding the pattern of violence over time and space provided by each data source. And lastly, thanks to the work of the the Humanitarian Law Center ${ }^{21}$, this estimate has recently been largely corroborated. Their attempt to generate an exhaustive list of victims has documented 9,030 murders and 1,200 missing from this time period.

\subsection{Peru}

Between 1980 and 2000, Peru witnessed a bloody armed internal conflict that was primarily carried out between the state forces and the revolutionary Communist Party of Peru-Sendero Luminoso (PCP-SLU) movement. This fighting received only limited attention from the international community.

Prior to the establishment of the Truth and Reconciliation Commission (Comision de la Verdad y Reconciliación, CVR), conventional wisdom had situated the number of victims claimed by the conflict to be approximately 25,000 . Using three different lists enumerating deaths and disappearances in Peru between 1980 and 2000, researchers at the CVR and the American Association for the Advancement of Science (AAAS) were able to conduct MSE analyses, which revealed that the total number of victims was in the vicinity of 70,000 (see Ball et al. (2003)).

The CVR collected reports documenting deaths and disappearances of approximately 24,000 people, of which 18,397 could be identified sufficiently to be matched with the other lists. Apart from the CVR list, the data collected by the Ombudsman Office (Defensoria del Pueblo, DP) formed the second list, and the third list comprised of a combination of data collected by several non-governmental organizations: the National Coalition of Human Rights (CNDDHH), the Agricultural Development Center (CEDAP), the Human Rights Commission (COMISEDH) and the International Committee of the Red Cross (ICRC). The CVR accounts for the majority of cases, as the addition of the second and third list only amounted to approximately 6,000 more cases (a total of 24,692). It is important to mention this, as it exemplifies the fact that the size of the additional lists used for MSE is less important than the pattern of overlap between the lists. Despite almost $75 \%$ of all reported cases having been recorded by the CVR, the two lists added for MSE delivered the missing information that was required to calculate an estimate.

Local area experts expected incidences to be reported with varying probability in differ-

\footnotetext{
${ }^{21}$ www.hlc-rdc.org/index $\cdot$ php?lid=en\&show=kosovo\&action=search\&str_stanje=1
} 
ent regions (i.e., violations of the homogeneity assumption), so data was first stratified by geographic location of the death or disappearance. Depending on the amount of information available for each region, the data was stratified by departments, provinces and - where possible even districts. For example, in the department of Ayacucho, the data could be stratified right down to the district-level, as all three lists had recorded a disproportionate number of incidences in this department. Besides the assumption that different regions would produce heterogeneous capture probabilities, it was assumed that the perpetrator by whom an individual was killed or disappeared would have an influence on whether the incident was reported or not. As demonstrated in Landman and Gohdes (this volume), the three lists offered a very different answer to the question which perpetrator should be held responsible for the majority of atrocities committed. Of all cases attributed to PCP-SLU, 80\% were exclusively recorded by the CVR database. For each geographical stratum, the researchers thus attempted to calculate individual estimates for the different perpetrators.

The log-linear models used for the estimation allowed for the modeling of interactions between the different lists, enabling the researchers to select the best fitting model out of seven possible models for each stratum. ${ }^{22}$ The models that were selected with the greatest frequency were those in which there was at least one interaction between the two smaller lists, with the Truth Commission's list being independent. Accordingly, not once was a model selected that assumed an interaction between the Truth Commission's data and both of the other lists.

With the help of this method, it could not only be revealed that the majority of atrocities were actually committed by the PCP-SLU (31,331, 95\% confidence interval:[24,823; 37,840]) and not by the state $(20,458,95 \%$ ci:[17,023; 23,893$])$, as had been assumed by many human rights groups (the data they had collected supported their claim), but that the conflict had primarily affected the rural, poor areas of Peru, furthest away from the urban agglomeration of Lima. Of the estimated 69,280 (95\% ci:[61,007; 77,552]), deaths and disappearances, 26,259 could be attributed to the region of Ayacucho alone, which is located in the south-central Andes.

It is important to take into consideration in this case that, despite its horrifying magnitude, the total death toll directly attributable to the conflict represents a minute fraction of the total Peruvian population at the time - approximately 27 million in 1993 . This means that other, more traditional, techniques of assessing conflict-related mortality rates, such as survey sampling, might have been unfeasible due to the low prevalence of the effects we would

\footnotetext{
${ }^{22}$ The seven models included one model that assumes independence between the lists, three that assume one interaction (i.e. two lists are dependent), and three that assumed two interactions (i.e. one list interacts with the other two lists, but the other two lists are independent of each other).
} 
need to detect (see (Silva and Ball, 2008)).

\section{Conclusion}

Multiple Systems Estimation methods encompass a broad variety of techniques that offer promising solutions to some of the challenges that researchers and practitioners face in casualty estimation in conflict and post-conflict situations. In the demanding circumstances of wartorn regions, obtaining reliable estimates of killed and disappeared persons poses a difficult, and sometimes seemingly impossible task. Documentation of violent events is often rare and biased, and the lack of infrastructure and resources presents a challenging situation for the conduct of surveys. MSE techniques offer a way to use existing, sometimes unrepresentative, information on casualties to produce a less biased and more complete number of atrocities committed. While certainly not a 'foolproof' class of methods, our case studies of Kosovo and Peru illustrate that in certain situations, it can considerably improve our knowledge of conflict trajectories.

In this paper, we have attempted to present the general intuition that lies behind MSE methods. Instead of focusing on one particular technique, we introduced a general framework for MSE analysis in order to explore more deeply the assumptions and the subtle interplay between heterogeneity and dependence. As with any other statistical technique, the most basic forms of MSE rely on strong assumptions which, in real life applications such as casualty estimation, are almost never met. Fortunately, the availability of more than two lists makes it possible to apply methods that replace those assumptions with more appropriate ones. Furthermore, multiple lists make it possible to test for violations of many of the assumptions implied by different models.

As illustrated in our case studies, researchers must examine the data and choose appropriate methods. This requires both statistical and local area expertise, as contextual knowledge about the data can guide researchers in terms of which assumptions are likely to be violated and which tests to check. At the same time, the appropriate method not only can reveal which assumptions are not met, but also it can help account for these violations.

The Kosovarian and Peruvian cases presented here exemplify the significance of data analysis methods that correct for recording biases in casualty estimation. In both situations, the number of casualties additionally 'uncovered' through MSE were larger than the number of killed and disappeared people recorded by Truth Commissions, NGOs and international organizations together. The political relevance of such results is evident, and illustrates the 
importance of comprehending the assumptions and possible pitfalls of such estimation techniques.

The many advantages of MSE come at a price of high technical complexity. As we have shown, MSE methods are substantially different from many more common statistical techniques, and their correct application require considerable expertise. Moreover, this complexity can create a communication barrier, preventing the clear dissemination and discussion of results, even with moderately sophisticated audiences. Opaque presentations, coupled with the potential misunderstanding of the methods' assumptions and the unavoidable analysis decisions, run the risk of undermining the credibility of otherwise sound conclusions. This can be particularly problematic in - as is almost always the case in a casualty-estimation context - a politically charged debate, if not addressed appropriately.

Since their first development for the estimation of wildlife populations over a century ago, recapture methods have significantly progressed. The recent evolvement of techniques that address 'real-life' problems, such as the estimation of casualties, presents an important step in the continuing development of this class of methods. Although they do present challenges and limitations, we believe that MSE methods are a versatile tool that enables the principled use of data frequently found in practice, and as such should be considered part of a standard 'casualty-estimation toolbox'. 


\section{References}

Ball, Patrick, Jana Asher, David Sulmont and Daniel Manrique. 2003. "How Many Peruvians Have Died? An Estimate of the Total Number of Victims Killed or Disappeared in the Armed Internal Conflict Between 1980 and 2000." Washington, DC: Report to the Peruvian Commission for Truth and Justice (CVR).

Ball, Patrick, Wendy Betts, Fritz Scheuren, Jana Dudukovich and Jana Asher. 2002. "Killings and Refugee Flow in Kosovo March - June 1999." Washington, DC: A Report to the International Criminal Tribunal for the Former Yugoslavia.

Basu, S. and N. Ebrahimi. 2001. "Bayesian capture-recapture methods for error detection and estimation of population size: Heterogeneity and dependence." Biometrika 88:269-279.

Bishop, Yvonne M. M., Stephen Fienberg and Paul H. Holland. 1975. Discrete Multivariate Analysis: Theory and Practice. Cambridge, MA: MIT Press.

Darroch, John, Stephen Fienberg, Gary Glonek and Brian Junker. 1993. "A Three-Sample Multiple-Recapture Approach to Census Population Estimation with Heterogeneous Catchability." Journal of the American Statistical Association 88(423):1137-1148.

Fienberg, S. 1972. "The Multiple recapture census for closed populations and incomplete $2^{\wedge} \mathrm{k}$ contingency tables." Biometrika 59:591-603.

Fienberg, Stephen E., Matthew S. Johnson and Brian W. Junker. 1999. "Classical Multilevel and Bayesian Approaches to Population Size Estimation Using Multiple Lists." Journal of the Royal Statistical Society. Series A (Statistics in Society) 162(3):pp. 383-405.

URL: http://www.jstor.org/stable/2680485

for Disease Monitoring, International Working Group and Forecasting. 1995a. "Capturerecapture and multiple-record systems estimation I: History and theoretical develop- ment." American Journal of Epidemiology, 142:1047-1058.

for Disease Monitoring, International Working Group and Forecasting. 1995b. "Capturerecapture and multiple-record systems estimation II: Applications in human diseases." American Journal of Epidemiology 142:1059-1068.

George, E. and C. Robert. 1992. "Capture-Recapture Estimation Via Gibbs Sampling." Biometrika p. 677-683.

Goudie, I. and M. Goudie. 2007. "Who captures the marks for the Petersen estimator?" Journal of the Royal Statistical Society. Series A 170:825-839. 
Hook, E. and R. Regal. 1999. "Recommendations for presentation and evaluation of capturerecapture estimates in epidemiology." Journal of clinical epidemiology, 52:917.

Lum, K., M. Price, T. Guberek and P. Ball. 2010. "Measuring Elusive Populations with Bayesian Model Averaging for Multiple Systems Estimation: A Case Study on Lethal Violations in Casanare, 1998-2007." Statistics, Politics, and Policy 1(1):2.

Manrique-Vallier, Daniel and Stephen E. Fienberg. 2008. "Population Size Estimation Using Individual Level Mixture Models." Biometrical Journal 50(6):1-13.

Petersen, C. 1896. "The yearly immigration of young plaice into the Limfjord from the German Sea." Report of the Danish Biological Station 6:1-48.

Sekar, Chandra C. and Edwards W. Deming. 1949. "On a Method of Estimating Birth and Death Rates and the Extent of Registration." Journal of the American Statistical Association 44(245):101-115.

Silva, Romesh and Patrick Ball. 2008. "The Demography of Conflict-Related Mortality in Timor-Leste (1974-1999): Reflections on Empirical Quantitative Measurement of Civilian Killings, Disappearances, and Famine-Related Deaths." Statistical Methods for Human Rights p. 117. 